\title{
Use of ginseng to reduce post-myocardial adverse myocardial remodeling: applying scientific principles to the use of herbal therapies
}

\author{
Sreedhar Bodiga • Wang Wang • Gavin Y. Oudit
}

Published online: 22 February 2011

(C) Springer-Verlag 2011

Cardiac remodeling involves molecular, cellular, and interstitial changes that manifest clinically as changes in size, shape, and function of the heart after injury or stress stimulation and was initially coined to describe the prominent changes that occur after myocardial infarction (MI) [1]. Adverse ventricular remodeling during the healing phase after acute MI continues to be an important problem that impacts adult cardiology practice. Indeed, coronary artery disease is now the most common cause of heart failure in westernized countries and is rising in prevalence in India and China. Most clinicians and cardiovascular researchers recognize that significant left ventricular (LV) remodeling occurs during infarct healing, and optimal healing is critical for survival with a favorable outcome. Over the last three decades, several laboratories have been actively searching for specific molecular targets that may lead to the development of therapies and strategies to optimize postinfarct healing, prevent adverse remodeling, and improve clinical outcome. However, specific therapy to optimize healing and prevent adverse post-MI remodeling

Clinical implications: Yin H et al. Ginsenoside-Rg1 enhances angiogenesis and ameliorates ventricular remodeling in a rat model of myocardial infarction.

S. Bodiga $\cdot$ G. Y. Oudit $(\bowtie)$

Division of Cardiology, Department of Medicine,

Mazankowski Alberta Heart Institute, University of Alberta,

Edmonton, Alberta T6G 2S2, Canada

e-mail: gavin.oudit@ualberta.ca

S. Bodiga $\cdot$ W. Wang $\cdot$ G. Y. Oudit

Mazankowski Alberta Heart Institute, University of Alberta,

Edmonton, Canada

W. Wang $\cdot$ G. Y. Oudit

Department of Physiology, University of Alberta,

Edmonton, Canada is currently lacking and only suboptimal medical therapy currently exists. Hearts continue to enlarge after MI, and heart failure is a growing burden. At present, ginseng is one of the most extensively used alternative medications throughout the world and has appeared in the pharmacopoeias of several western countries, including the United States and European countries with indications for cardiovascular diseases and other conditions [2].

In this issue, Yin $H$ et al. showed that the active ingredient of the ginseng extract, ginsenoside- $\operatorname{Rg} 1$, enhances angiogenesis and reduces adverse ventricular remodeling in a rat model of MI [3]. Ginsenoside-Rg1 is the most prevalent and active constituent of ginseng. Three major targets of adverse myocardial remodeling were the key mediators of the cardioprotective effects seen with ginsenoside-Rg1administration: stimulation of the PI3K/ Akt [and inhibition of p38 mitogen-activated protein kinase (MAPK)] pathway with improvement in post-MI angiogenesis and reduction in myocardial fibrosis (Fig. 1). These beneficial effects resulted in reduced LV dilation and a modest improvement in systolic function based on the assessment of fractional shortening and ejection fraction. PI3K inhibition using LY294002 also reduced the phosphorylation of the p38 MAPK pathway which is known to play a key role in inducing inflammatory cytokines and apoptotic cell death. The PI3K/Akt pathways mediate cardioprotective effects in a wide variety of pathological conditions including important anti-apoptotic effects and modulation of cellular growth and metabolism [4]. However, pharmacological inhibition of PI3K signaling using LY294002 cannot distinguish isoform-specific effects of PI3K signaling which is well established to play a differential role in myocardial ischemic injury [4]. In addition, LY294002 is a potent blocker of voltage-gated $\mathrm{K}^{+}$channels in cardiomyocytes [5]. Thus, off-target effects 


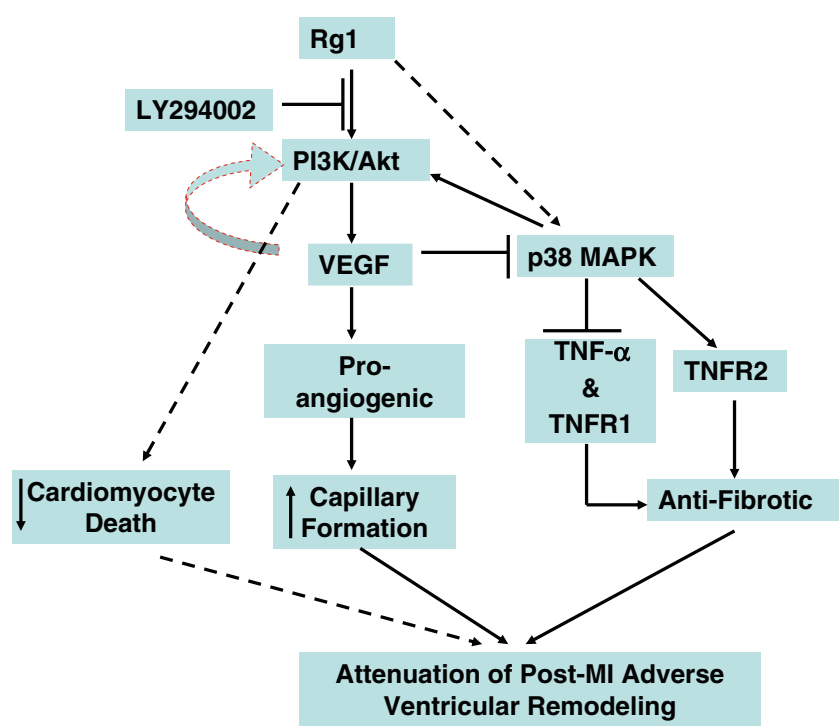

Fig. 1 A schematic representation of the effects of ginsenoside-Rg1 on myocardial signaling, inflammation, angiogenesis, and fibrosis resulting in the attenuation of adverse remodeling of the postmyocardial infarcted myocardium. Ginsenoside-Rg1 enhanced VEGF-induced angiogenesis and attenuated TNF- $\alpha$-induced myocardial fibrosis via the activation of PI3K/Akt signaling, inhibiting p38 MAPK and crosstalk between the two signaling pathways. Solid lines indicate established effects, whereas dashed lines represent putative mechanisms

of this drug must be considered when interpreting its action on the myocardium. Clearly, more studies are needed to further delineate the specific PI3K isoform responsible for the beneficial effects of ginsenoside-Rg1 on Akt activation and other key mediators of cardioprotection such as GSK $3 \beta$. In addition, infiltration of neutrophils and macrophages into the post-infarcted myocardium and the subsequent inflammation is controlled by the PI3K $\gamma$ pathway and PI3K inhibition is expected to alter this inflammatory response.

Yin et al. [3] showed that administration of ginsenosideRg1 increased vascular endothelial growth factor (VEGF) expression levels in vivo and in cultured human umbilical vein endothelial cells. The upregulation of VEGF resulted in increased density of newly formed vessels in the postinfarcted myocardium [3]. Activation of PI3K/Akt signaling is a well-known mediator of angiogenesis and represents a crosstalk between the coronary endothelium and the cardiomyocyte compartment [4]. These results are consistent with previous findings showing that ginsenoside- $\operatorname{Rg} 1$ is a potent proangiogenic factor and may potentially be useful in therapeutic angiogenesis [6]. Phosphatase and tensin homolog (PTEN) is a 3'-lipid phosphatase and a critical negative regulator of PI3K signaling in the heart. Upregulation in PI3K signaling may also be triggered by a reduction in PTEN activity [4]. As such, future studies should examine the effects of ginsenoside-Rg1on PTEN expression and activity.

Inflammation and extracellular matrix remodeling are two key components of healing and LV remodeling after MI [7-9]. Several pro-inflammatory cytokines, including tumor necrosis factor (TNF) $\alpha$, are consistently upregulated in the infarct zone after experimental MI. In their work, Yin et al. [3] showed in post-infarcted rat myocardium the upregulation of TNF $\alpha$ and tumor necrosis factor receptor (TNFR)1 levels was drastically reduced while TNFR2 levels were increased in response to treatment with ginsenoside- $\operatorname{Rg} 1$. However, the paradoxical clinical trial results with TNF $\alpha$ blockers implied a more complicated role for TNF $\alpha$ in heart failure than currently considered. Indeed, TNF $\alpha$ mediated effects are not uniformly detrimental in the heart. As a stress-response protein, TNF $\alpha$ can be cytoprotective during conditions such as ischemic injury and coronary microembolization. TNF $\alpha$ signaling occurs via two cellsurface receptors (TNFR1 and TNFR2) and in large part via the TNFR-associated factor 2 (TRAF2)-dependent activation of nuclear factor (NF)- $\mathrm{kB}$, p38 MAPK, and c-Jun Nterminal kinase. TNFR1 and TNFR2 have disparate and opposing effects on remodeling, hypertrophy, NF- $\mathrm{kB}$, inflammation, and apoptosis in heart failure: TNFR1 exacerbates, whereas TNFR2 ameliorates these events. Signaling through both receptors is required to induce diastolic dysfunction and oxidative stress [10]. Moreover, in addition to the intrinsically complex TNF $\alpha / T N F R 1 /$ TNFR2 signaling, membrane-bound TNF $\alpha$, and/or TNFR1/TNFR2 complex is processed by TNF-alpha converting enzyme or ADAM17, a process inhibited by tissue inhibitor of matrix metalloproteinase-3 [7]. TNF $\alpha$ induced upregulation of matrix metalloproteinases in cardiomyocytes and cardiofibroblasts is another critical link between inflammation and adverse remodeling of the extracellular matrix [11].

The assessment of myocardial fibrosis was based on a collection of standard histological techniques and mRNA expression and protein levels of collagen I. Collagen I is the most abundant fibrillar collagen in the heart and is a major determinant of myocardial stiffness. Treatment with ginsenoside reduced the degree of fibrosis and collagen I expression, a process partly dependent on intact PI3K signaling [3]. Diastolic dysfunction in the post-MI setting is an important determinant of clinical outcomes. Although, Yin et al. did not assess diastolic function in their study [3], reduction in myocardial fibrosis is expected to reduce myocardial stiffness and improve post-MI induced diastolic dysfunction. The specific effects of ginsenoside on cardiofibroblasts and cardiomyocytes need to be examined using a culture-based system since the crosstalk between these 
major cell types in the heart is a critical determinant of the extent and degree of myocardial fibrosis.

Despite a significant improvement in LV function, ginsenoside-Rg1 treatment did not completely halt the progression of LV remodeling. In addition to preventing the degree of LV dilation and preserving systolic function, the molecular and cellular changes mediated by ginsenoside-Rg1 may have also resulted in reduced postMI LV rupture, an important cause of mortality in the modern era [12]. Importantly, while the overall mortality was $10.4 \%$, no animal died from LV rupture. Adverse electrical remodeling characterized by downregulation of voltage-gated $\mathrm{K}^{+}$channels and action potential prolongation are key drivers of the ventricular arrhythmias and sudden cardiac death in the post-MI setting. It is important to examine the impact of any potential new therapy on the post-MI electrical properties of the heart. As we move ahead, we need to better define the pharmacokinetics and pharmacodynamics of ginsenoside-Rg1. For example, what were the therapeutic plasma and tissue levels obtained with the daily intraperitoneal administration of ginsenoside-Rg1 in rats? Can this be obtained in patients using the galentical preparation (ginseng root) or by high dose consumption of purified ginsenoside-Rg1? The precise molecular and cellular mechanism of ginsenoside-Rg1 action also remains to be better defined. Metabolomic profiling of stressed rats treated with ginseng extracts showed that several major metabolic pathways including catecholamines, glucocorticoids, and the tricarboxylic acid cycle are altered resulting in metabolic regulation and compensation [13]. A systems biology approach may be a useful strategy to identify novel targets of ginsenoside- $\operatorname{Rg} 1$. The potent antioxidant effects of ginsenoside-Rg1 on isolated cardiomyocytes may also confer benefits to the post-infarcted myocardium.

The findings from the Yin $\mathrm{H}$ et al. study support a clear benefit of ginsenoside-Rg1 on post-MI adverse myocardial remodeling. Several galentical preparations have yielded important drugs for the treatment of cardiovascular diseases. These include the chemical modification of salicylic acid derived from the willow bark to generate acetylsalicylic acid, digoxin, a purified cardiac glycoside extracted from the foxglove plant, Digitalis lanata, and reserpine isolated from the dried root of Rauwolfia serpentina. Contrary to popular belief, the use of herbal remedies can pose serious health risks. Besides the direct risks of adverse effects and drug interactions, there is an indirect risk that an herbal remedy without demonstrated efficacy may compromise, delay, or replace an effective form of conventional treatment [2]. The use of the Asian ginseng root for various indications has shown unclear efficacy for each indication and highlights the need for additional randomized con- trolled trials [2]. Clearly, more scientific-sound studies are need as we continue to explore the potential therapeutic benefits of herbal therapies including the use of ginsenoside-Rg1 as potential new therapies for cardiovascular disease. Moreover, any new therapy must be tested against conventional therapies including ACE inhibitor, beta-adrenergic receptor blocker, and aldosterone receptor blocker which have all been shown to reduce adverse postMI remodeling and improve clinical outcomes.

Acknowledgements GYO is a Clinician-Investigator of the Alberta Innovates - Health Solutions and the Distinguish Clinician Scientist of the Heart and Stroke Foundation of Canada and Canadian Institutes of Health Research. SB is a Post-Doctoral Fellow of Alberta Innovates Health Solutions and WW is supported by a Mazankowski Alberta Heart Institute Studentship.

\section{References}

1. Pfeffer MA, Braunwald E (1990) Ventricular remodeling after myocardial infarction. Experimental observations and clinical implications. Circulation 81:1161-1172

2. De Smet PA (2002) Herbal remedies. N Engl J Med 347:2046-2056

3. Yin H, Liu Z, Li F, Ni M, Wang B, Qiao Y, Xu X, Zhang M, Zhang J, Lu H, Zhang Y (2011) Ginsenoside-Rg1 enhances angiogenesis and ameliorates ventricular remodeling in a rat model of myocardial infarction. J Molecular Medicine (in press)

4. Oudit GY, Penninger JM (2009) Cardiac regulation by phosphoinositide 3-kinases and PTEN. Cardiovasc Res 82:250-260

5. Sun H, Oudit GY, Ramirez RJ, Costantini D, Backx PH (2004) The phosphoinositide 3-kinase inhibitor LY294002 enhances cardiac myocyte contractility via a direct inhibition of Ik, slow currents. Cardiovasc Res 62:509-520

6. Leung KW, Pon YL, Wong RN, Wong AS (2006) Ginsenoside$\operatorname{Rg} 1$ induces vascular endothelial growth factor expression through the glucocorticoid receptor-related phosphatidylinositol 3-kinase/Akt and beta-catenin/T-cell factor-dependent pathway in human endothelial cells. J Biol Chem 281:36280-36288

7. Kassiri Z, Khokha R (2005) Myocardial extra-cellular matrix and its regulation by metalloproteinases and their inhibitors. Thromb Haemost 93:212-219

8. Jugdutt BI (2010) Preventing adverse remodeling and rupture during healing after myocardial infarction in mice and humans. Circulation 122:103-105

9. Kandalam V, Basu R, Abraham T, Wang X, Soloway PD, Jaworski DM, Oudit GY, Kassiri Z (2010) TIMP2 deficiency accelerates adverse post-myocardial infarction remodeling because of enhanced MT1-MMP activity despite lack of MMP2 activation. Circ Res 106:796-808

10. Hamid T, Gu Y, Ortines RV, Bhattacharya C, Wang G, Xuan YT, Prabhu SD (2009) Divergent tumor necrosis factor receptorrelated remodeling responses in heart failure: role of nuclear factor-kappaB and inflammatory activation. Circulation 119:1386-1397

11. Awad AE, Kandalam V, Chakrabarti S, Wang X, Penninger JM, Davidge ST, Oudit GY, Kassiri Z (2009) Tumor necrosis factor induces matrix metalloproteinases in cardiomyocytes and cardiofi- 
broblasts differentially via superoxide production in a PI3Kgammadependent manner. Am J Physiol Cell Physiol 298:C679-C692

12. Pouleur AC, Barkoudah E, Uno H, Skali H, Finn PV, Zelenkofske SL, Belenkov YN, Mareev V, Velazquez EJ, Rouleau JL, Maggioni AP, Kober L, Califf RM, McMurray JJ, Pfeffer MA, Solomon SD (2010) Pathogenesis of sudden unexpected death in a clinical trial of patients with myocardial infarction and left ventricular dysfunction, heart failure, or both. Circulation 122:597-602

13. Wang M, Lamers RJ, Korthout HA, van Nesselrooij JH, Witkamp RF, van der Heijden R, Voshol PJ, Havekes LM, Verpoorte R, van der Greef J (2005) Metabolomics in the context of systems biology: bridging traditional Chinese medicine and molecular pharmacology. Phytother Res 19:173-182 